\title{
Unconventional magnetism as a consequence of the charge disproportionation and the molecular orbital formation in $\mathrm{Ba}_{4} \mathrm{Ru}_{3} \mathrm{O}_{10}$
}

\author{
S.V. Streltsov ${ }^{1,2}$ and D.I. Khomskii ${ }^{3}$ \\ ${ }^{1}$ Institute of Metal Physics, S.Kovalevskoy St. 18, 620990 Ekaterinburg, Russia \\ ${ }^{2}$ Ural Federal University, Mira St. 19, 620002 Ekaterinburg, Russid* \\ ${ }^{3}$ II. Physikalisches Institut, Universität zu Köln, Zülpicher Straße 77, D-50937 Köln, Germany
}

(Dated: November 6, 2018)

\begin{abstract}
The magnetic and electronic properties of $\mathrm{Ba}_{4} \mathrm{Ru}_{3} \mathrm{O}_{10}$ were investigated by the ab-initio and model calculations. It is shown that nonmagnetic ground state of the one-third $\mathrm{Ru}^{4+}$ ions is not due to the correlation effects. It is rather caused by the charge disproportionation between crystallographically different $\mathrm{Ru}$ and the molecular orbital formation in the Ru's trimer.
\end{abstract}

PACS numbers: 75.47.Lx, 75.50.Ee, 71.27.+a

\section{INTRODUCTION}

The complex $\mathrm{Ru}$ oxides are widely investigated in the last decades because of their unusual electronic and magnetic properties. The odd-parity pairing was found in the superconductor $\mathrm{Sr}_{2} \mathrm{RuO}_{4}$, 1] while $\mathrm{Ca}_{2} \mathrm{RuO}_{4}$ became one of the model systems for study of the orbital-selective Mott transition. 2] The integer spins on the $\mathrm{Ru}^{4+}$ ions likely form the Haldane chains in $\mathrm{Tl}_{2} \mathrm{Ru}_{2} \mathrm{O}_{7}$, which leads to a drastic decrease of the magnetic susceptibility at low temperatures. [3] Similar behavior of susceptibility was recently observed in another $\mathrm{Ru}$ oxide $-\mathrm{Ba}_{4} \mathrm{Ru}_{3} \mathrm{O}_{10}$. [4] This drastic decrease was initially attributed to the formation of the unconventional antiferromagnetic (AFM) state, but the mechanism of its stabilization is still unknown.

Structurally $\mathrm{Ba}_{4} \mathrm{Ru}_{3} \mathrm{O}_{10}$ is made of the $\mathrm{Ru}$-trimers. These trimers are formed by three $\mathrm{RuO}_{6}$ octahedra sharing their faces. Each trimer is connected with four neighboring trimers (see Fig. 1) via corners of the outer $\mathrm{RuO}_{6}$ octahedra to build corrugated layers. We denote the middle $\mathrm{Ru}$ ion in the trimer $\mathrm{Ru}_{m}$, while two outer $\mathrm{Ru}-\mathrm{Ru}_{o}$.

In $\mathrm{Ba}_{4} \mathrm{Ru}_{3} \mathrm{O}_{10}$ the $\mathrm{Ru}$ ions must have $4+$ oxidation state with 4 electrons in the $4 d$-shell, since $\mathrm{Ba}$ is $2+$ and $\mathrm{O}$ is $2-$. Due to a large $t_{2 g}-e_{g}$ crystal-field splitting (see Sec. III for details) these four $4 d$-electrons should be stabilized in the $t_{2 g}$ sub-shell resulting in the state with $\mathrm{S}=1$. Indeed, at room temperature $\mathrm{Ba}_{4} \mathrm{Ru}_{3} \mathrm{O}_{10}$ was found to be paramagnetic with the effective moment $\mu_{\text {eff }}=2.83 \mu_{B}$ typical for the $S=1$ ions, [4, 5] while the decrease of the temperature below $T_{N}=105 \mathrm{~K}$ leads to the formation of the unconventional AFM state, where one-third of the $\mathrm{Ru}^{4+}$ ions are not ordered (tentatively these are $\mathrm{Ru}_{m}$ ). [4]

Several models were proposed in Ref. 4 to explain this physical phenomenon. One may think that (1) due to some reason a part of the $\mathrm{Ru}$ ions stays paramagnetic down to a very low temperature; that (2) they are randomly frozen; or that (3) because of a strong spin-orbit

*Electronic address: streltsov@imp.uran.ru coupling a nonmagnetic state of the $\mathrm{Ru}$ ion with $J=0$ is stabilized.

In order to check all these possibilities, the ab-initio band structure calculations were performed for this compound. We found that the true reason for the absence of the magnetic moments on one-third of the $\mathrm{Ru}$ ions is the charge disproportionation between two crystallographically nonequivalent $\mathrm{Ru}\left(\mathrm{Ru}_{m}\right.$ and $\left.\mathrm{Ru}_{o}\right)$ and formation of the molecular orbital in the trimers. In the bandstructure picture it is manifested in the suppression of the nonmagnetic $4 \mathrm{~d}$ density of states (DOS) of the middle $\mathrm{Ru}_{m}$ in the vicinity of the Fermi level.

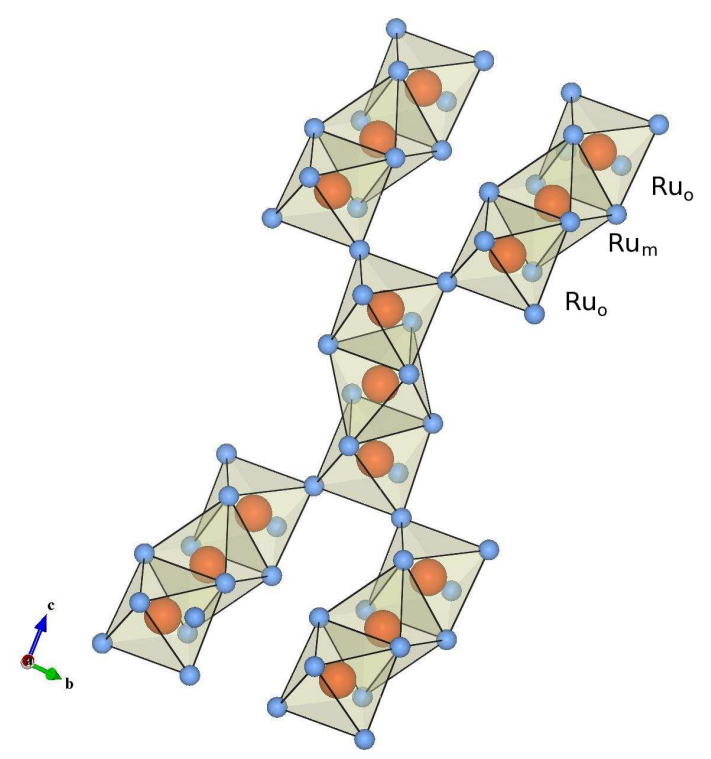

FIG. 1: (color online). The crystal structure of $\mathrm{Ba}_{4} \mathrm{Ru}_{3} \mathrm{O}_{10}$. Projection on the $b c-$ plane. Oxygen ions are in blue, Ruthenium - in red, $\mathrm{Ba}$ - not shown for the simplicity. $\mathrm{Ru}_{m}$ are in the middle of the trimers, $\mathrm{Ru}_{o}-$ on the corners. 


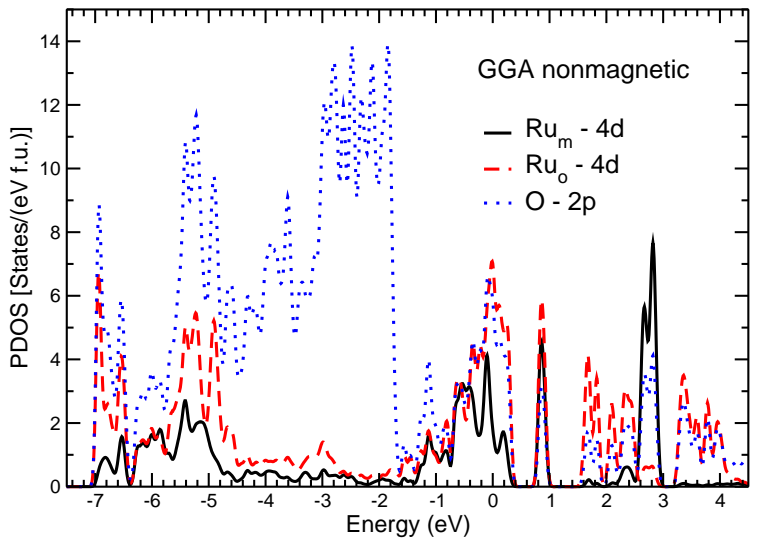

FIG. 2: (color online). The GGA partial density of states, obtained in the PWscf code. $\mathrm{Ru}_{m}$ is the middle atom in the trimer, while $\mathrm{Ru}_{o}$ are the corner, outer to the trimers, ones. The Fermi energy corresponds to zero.

\section{CALCULATION DETAILS}

For the band structure calculations we primary used the pseudo-potential PWscf code. [6] We utilized generalized gradient approximation (GGA) with Perdew-BurkeErnzerhof version of the exchange-correlation potential [7] and ultrasoft scalar-relativistic pseudo-potentials with nonlinear core correction (for better description of the magnetic interactions). The charge density and kinetic energy cut-offs were taken $40 \mathrm{Ry}$ and $200 \mathrm{Ry}$, respectively. $664 k$-points in a full Brillouin zone were used in the calculation.

The generalized gradient approximation with the account of the on-site Coulomb repulsion (GGA+U method) 8] was used to test an importance of the electronic correlations. The Hubbard's on-site repulsion parameter $U$ and Hund's rule intra-atomic exchange $J_{H}$ for $\mathrm{Ru}$ were calculated in Ref. 3 to be $3.0 \mathrm{eV}$ and $0.7 \mathrm{eV}$.

The Wannier function projection was performed within the linearized muffin-tin orbitals (LMTO) method [9] as it was described in Ref. 10.

All the calculations were performed for the crystal structure corresponding to $\mathrm{T}=10 \mathrm{~K}$. [4]

\section{NONMAGNETIC GGA CALCULATIONS}

We start with the analysis of the results obtained in the nonmagnetic generalized gradient approximation (GGA), presented in Fig. 2. One may see that the oxygen $2 \mathrm{p}$ states are placed primarily from -7 to approximately $1 \mathrm{eV}$. The top of the valence band is formed by Ru-4d $\left(t_{2 g}\right)$ states. The $\mathrm{Ru}-e_{g}$ orbitals are above $1.5 \mathrm{eV}$. The $t_{2 g}-e_{g}$ crystal field splitting estimated by the center of

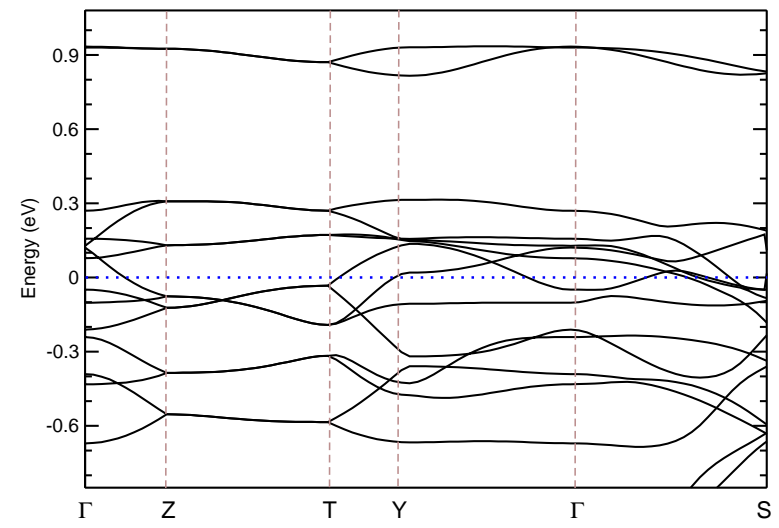

FIG. 3: (color online). The band structure obtained in the nonmagnetic GGA calculations (in the PWscf code). The Fermi energy corresponds to zero.

gravity calculation is 3.38 and $3.20 \mathrm{eV}$ for $\mathrm{Ru}_{m}$ and $\mathrm{Ru}_{o}$ respectively. Thus one would indeed expect that such a strong crystal field splitting would prevent occupation of the $e_{g}$ orbitals and $d^{4}$ configuration of $\mathrm{Ru}^{4+}$ would result in the $\mathrm{S}=1$ ground state of each $\mathrm{Ru}$ ion.

However, there is another one quite important feature of the nonmagnetic DOS related to the splitting between the $\mathrm{Ru}_{m}$ and $\mathrm{Ru}_{o}$ 4d states, which is clearly seen from Fig. 2. The same center of gravity calculation shows that this splitting exceeds $0.13 \mathrm{eV}$. The $\mathrm{Ru}_{m}-4 \mathrm{~d}$ states integrally turns out to be lower than $\mathrm{Ru}_{o}-4 \mathrm{~d}$ due to a larger mean Ru-O distance. According to Ref. 4, the difference in the mean $\mathrm{Ru}-\mathrm{O}$ distances for these two $\mathrm{Ru}$ exceeds $0.035 \AA$. This is not a small number, since the difference in ionic radii between $\mathrm{Ru}^{4+}$ and $\mathrm{Ru}^{3+}$ is $0.06 \AA$. [11] As a result one could expect that there would be more $\mathrm{d}-$ electrons on the middle $\mathrm{Ru}$, i.e. it would have on the average a smaller valence. And indeed, according to our nonmagnetic GGA calculations the difference in charge between $\mathrm{Ru}_{m}$ and $\mathrm{Ru}_{o}$ is 0.52 electron per atom, which is even larger than the charge disproportionation on the Fe sites in the charge ordered phase of $\mathrm{Fe}_{3} \mathrm{O}_{4}$. [12]

Thus, $\mathrm{Ru}_{m}-t_{2 g}$ partial DOS states turns out to be almost completely below the Fermi level, except a small shoulder and a narrow peak at $\sim 0.9 \mathrm{eV}$. The detailed analysis of the band structure, presented in Fig. 3, confirms that the band corresponding to this peak in DOS at $0.9 \mathrm{eV}$ is dispersionless. One may also see that each band in the ZT direction is two times degenerate, since there are two formula units (f.u.) in the unit cell. In effect each trimer provides one dispersionless band at $\sim 0.9$ $\mathrm{eV}$.

In order to analyze the formation of this band we carried out the calculation within the local density approximation (LDA) and the LMTO method for which one may 


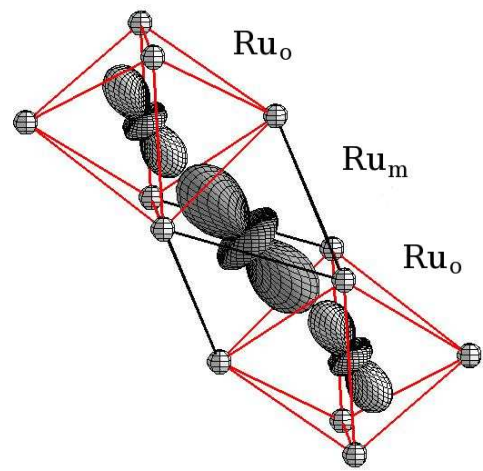

FIG. 4: (color online). The molecular orbital which corresponds to the highest in energy $\mathrm{Ru}-t_{2 g}$ states (bands at $\sim 0.9 \mathrm{eV}$ in Fig. 3), obtained by the diagonalization of the three-site Hamiltonian with the use of the Wannier function procedure in the LDA LMTO calculations.

perform the Wannier function projection procedure. 10] The use of this procedure allows to get the on-site Hamiltonian matrix for the $\mathrm{Ru}-4 d$ states and all the hoppings between different sites. The projection was performed onto thirty $\mathrm{Ru}-4 d$ orbitals $(5$ orbitals $\times 3$ atoms $\times 2$ formula units) in the local coordinate system, where the axis are directed to the nearest oxygens.

The diagonalization of the on-site Hamiltonian in a real space for each $\mathrm{Ru}$ leads to the order of the levels predicted by the local distortions. The tetragonal elongation of the $\mathrm{Ru}_{m} \mathrm{O}_{6}$ octahedra results in two low-lying almost degenerate $t_{2 g}$-levels split from the higher $t_{2 g}$ orbital by $\sim 330 \mathrm{meV}$. However, a close inspection of the Hamiltonian shows that the hopping integrals between $\mathrm{Ru}_{o}$ and $\mathrm{Ru}_{m}$ exceed $360 \mathrm{meV}$, which is, in contrast to naive expectations, even larger than the off-diagonal onsite matrix elements for the $t_{2 g}$ orbitals. Thus, the orbitals on which the $\mathrm{Ru}-4 d$ electrons tend to localize are determined not only by the crystal-field created by the local surroundings (oxygens), but also by the inter-site matrix elements. This is mainly due to a face-sharing packing of the $\mathrm{RuO}_{6}$ octahedra in the trimers, which allows a direct overlap between $d$-orbitals on the neighboring $\mathrm{Ru}$ sites. Indeed, the nearest neighbors $\mathrm{Ru}-\mathrm{Ru}$ distance across the common face $2.55 \AA$ is even less than that in ruthenium metal, $2.65 \AA$. [13] It is also quite important that the relevant orbitals here are $4 d$-orbitals, which are more extended than $3 d$. 14

In order to take into account the hoppings, we constructed the large $15 \times 15 \mathrm{Ru}_{o}-\mathrm{Ru}_{m}-\mathrm{Ru}_{o}$ Hamiltonian for the trimer in a real space. Diagonalizing this Hamiltonian one obtains that the lowest and highest in energy are the molecular orbitals which have the $a_{1 g}$ symmetry with the largest contribution on the central $\mathrm{Ru}_{m}$ ion. This is clearly seen in the Fig. 4, where the highest in energy antibonding $a_{1 g}^{*}$ molecular orbital is plotted. This orbital corresponds to the band, which has the energy $\sim 0.9 \mathrm{eV}$ in the $\mathrm{ZT}$ direction.

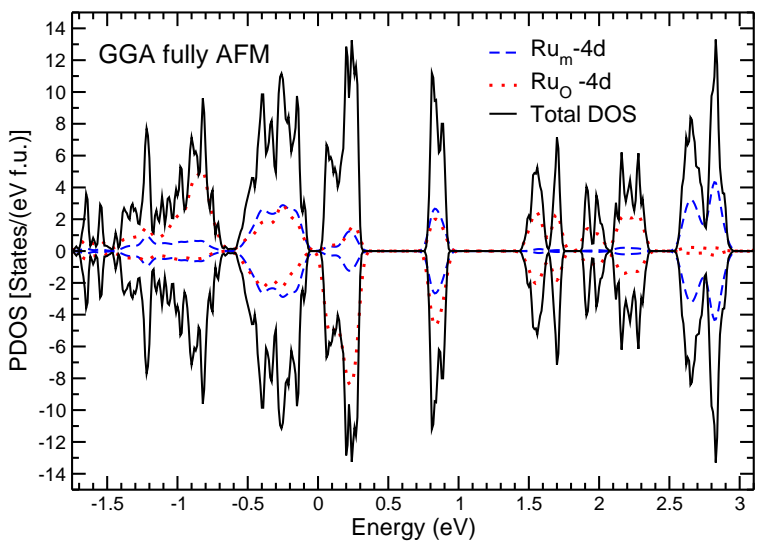

FIG. 5: (color online). The total and partial density of states (DOS) in the GGA calculation for the $\uparrow m \downarrow-\uparrow m \downarrow$ magnetic configuration (so called fully AFM), when the spins on the $\mathrm{Ru}_{o}$ both in the same and in the adjacent trimers are antiparallel. The results were obtained in the PWscf code. The Fermi energy corresponds to zero.

Inspecting Fig. 4 one may see that the contribution of the $\mathrm{Ru}_{m}-4 d$ states to the antibonding $a_{1 g}^{*}$ orbital should be the largest (the same is actually correct also for the bonding $a_{1 g}$ wave function). This is easy to understand by solving the three-site $(i=1,2,3)$ problem with the single orbital per site in the tight-binding approximation. For simplicity the energies of each orbital are taken the same, $\varepsilon_{i}=0$, and the hoppings, $t>0$, are nonzero only between the nearest sites. For the linear cluster of three atoms the Hamiltonian is written as

$$
H=\left(\begin{array}{lll}
0 & t & 0 \\
t & 0 & t \\
0 & t & 0
\end{array}\right) .
$$

The energy spectrum consists of the bonding (b), antibonding (ab) and nonbonding (nb) orbitals:

$$
\begin{gathered}
E_{a b}=t \sqrt{2}, \quad \psi_{a b}=\frac{1}{2}\left(\psi_{1}-\sqrt{2} \psi_{2}+\psi_{3}\right), \\
E_{n b}=0, \quad \psi_{n b}=\frac{1}{\sqrt{2}}\left(\psi_{1}-\psi_{3}\right), \\
E_{b}=-t \sqrt{2}, \quad \psi_{b}=\frac{1}{2}\left(\psi_{1}+\sqrt{2} \psi_{2}+\psi_{3}\right) .
\end{gathered}
$$

As a result one may see that the weight of the states corresponding to the middle, $i=2$, atom to the bonding and antibonding wave functions equals to $50 \%$, while they do not contribute to the nonbonding molecular orbital at all. Thus, the $\mathrm{Ru}_{m}-t_{2 g}$ states of the $a_{1 g}$ symmetry are pushed away from the Fermi level due to the molecular orbital formation. Moreover, the shift of the rest of the $\mathrm{Ru}_{m}-t_{2 g}$ states downwards, with the cor- 
TABLE I: Total energies, magnetic moments on the $\mathrm{Ru}_{o}$ and $\mathrm{Ru}_{m}$ ions and band gaps. Results of the GGA in the PWscf code. Total energies are per formula unit (f.u.). The notation $\uparrow m \downarrow$ means that two $\mathrm{Ru}_{o}$ ions are AFM, while moment for the $\mathrm{Ru}_{m}$ can be found in the 3rd column. Symbol "-" denotes the bond with adjacent trimers. The unit cell vectors are chosen in such a way, that the type of inter-trimer magnetic order (ferro- or antiferromagnetic) for presented configurations is the same for all 4 inter-trimer bonds.

\begin{tabular}{c|c|c|c}
\hline \hline & $\begin{array}{c}\text { Total energy } \\
(\mathrm{meV})\end{array}$ & $\begin{array}{c}\text { Spin moments } \\
\mathrm{Ru}_{o} / \mathrm{Ru}_{m}\left(\mu_{B}\right)\end{array}$ & $\begin{array}{c}\text { Band } \\
\text { gap }(\mathrm{eV})\end{array}$ \\
\hline$\uparrow m \downarrow-\uparrow m \downarrow$ & 0 & $0.95 / 0$ & 0.12 \\
$\uparrow m \uparrow-\uparrow m \uparrow$ & 22.7 & $0.98 / 0.33$ & Metal \\
$\uparrow m \downarrow-\downarrow m \uparrow$ & 20.1 & $0.94 / 0$ & Metal \\
$\uparrow m \uparrow-\downarrow m \downarrow$ & 61.0 & $0.69 / 0.15$ & Metal \\
$0 m 0-0 m 0$ & 137.6 & $0 / 0$ & Metal \\
\hline \hline
\end{tabular}

responding charge disproportionation, leads to the suppression of the $\mathrm{Ru}_{m} 4 d$ partial DOS in the vicinity of the Fermi level. The $\mathrm{Ru}_{o}-4 d$ states in contrast form well defined peak exactly at the Fermi energy.

The Stoner criteria cannot be applied directly to the partial DOS, but it is clear that the gain in the magnetic energy due to the spin splitting on $\mathrm{Ru}_{m}$ will be minimal (because of the pseudogap in its partial DOS), while the loss of the band energy will be substantial. Thus in the band picture the $\mathrm{Ru}_{m}-4 d$ states are not expected to be polarized. In contrast, the $\mathrm{Ru}_{o}-4 d$ states can be easily magnetized. This is exactly what we observe in the magnetic GGA calculations.

\section{MAGNETIC GGA CALCULATIONS}

Several configurations were investigated in our magnetic GGA calculations (see Tab. I). The lowest in energy is the state when the spins on the $\mathrm{Ru}_{o}$ both in the same and in the adjacent trimers are antiparallel $(\uparrow m \downarrow-\uparrow m \downarrow)$. The partial DOS corresponding to this configuration are plotted in Fig. 5. One may see that there is no splitting between majority and minority spins of the $\mathrm{Ru}_{m}$. Thus according to our calculation $1 / 3$ of the $\mathrm{Ru}$ atoms indeed are nonmagnetic, exactly as it was observed in the experiment. [4]

The magnetic moment on the $\mathrm{Ru}_{m}$ appears only in the configurations with the FM order of the $\mathrm{Ru}_{o}$ in the trimers. The ferromagnetically ordered spins of the $\mathrm{Ru}_{o}$ create an exchange field on the $\mathrm{Ru}_{m}$, which slightly magnetize these ions. On the band-structure language the appearance of some magnetization on $\mathrm{Ru}_{m}$ in a ferromagnetic state can be explained by the spin splitting of the $\mathrm{Ru}_{m}-4 d$ states due to their hybridization with the $\mathrm{Ru}_{o}-4 d$ states, which as we have shown above is large enough.

The magnetic moments on $\mathrm{Ru}_{o}$ are smaller than $2 \mu_{B}$ expected for $S=1$. This is a rather typical situation for the transition metal oxides (especially for those based on the $4 \mathrm{~d}$ and $5 \mathrm{~d}$ elements) and can be attributed to a strong hybridization with the oxygens. Because of the hybridization we also observe non zero magnetic moments on some of the oxygens, which vary from 0.04 to $0.26 \mu_{B}$, depending on the magnetic configuration and oxygens crystallographic positions. The largest magnetic moment, $0.26 \mu_{B}$, was found on the oxygen atoms, which belongs to two neighboring trimers in the fully FM solution ( $\uparrow \uparrow \uparrow \uparrow m \uparrow)$.

The analysis of the calculation results presented in Tab. Ishows that the magnetic contributions to the total energies of different solutions is not described by purely Heisenberg terms, like $J_{i j} \vec{S}_{i} \vec{S}_{j}$. Indeed, if one compares the total energies of two solutions with the AFM order in the trimer (e.g. $\uparrow m \downarrow-\uparrow m \downarrow$ and $\uparrow m \downarrow-\downarrow m \uparrow$ ) then the lowest will be the one with the AFM coupling between trimers. However, if one analyses another two solutions with the FM order in the trimer (e.g. $\uparrow m \uparrow-\uparrow m \uparrow$ and $\uparrow m \uparrow-\downarrow m \downarrow)$, it becomes clear that the lowest will be the one with the FM coupling between trimers. This is mostly related with the smaller magnetic moment on the $\mathrm{Ru}_{m}$ ions in the $\uparrow m \uparrow-\downarrow m \downarrow$ configuration, and it may be a consequence of molecular orbital formation on trimers".

There are also two other contributions to the magnetic energy in addition to the conventional Heisenberg model, which have to be taken into account to describe magnetic properties of $\mathrm{Ba}_{4} \mathrm{Ru}_{3} \mathrm{O}_{1} 0$. This is the development of the magnetic moments on $\mathrm{Ru}_{m}$ (when the spins in the trimer are parallel) and on those oxygens, which are shared by two trimers (when the spins on the neighbor trimers are FM ordered). In each case the contribution to the total energy is $-I M^{2} / 4$, where $M$ is the magnetic moment on the $\mathrm{Ru}_{m}$ or $\mathrm{O}$ ions, and $I$ - the Stoner parameter, which is approximately equals to the Hund's rule exchange parameter for $\mathrm{Ru}(0.7 \mathrm{eV})$ and to $1.6 \mathrm{eV}$, as it was calculated in Ref. [15]

It is important to mention that only fully AFM state, corresponding to the lowest total energy, is insulating in the GGA approach, without including electronic correlations (Hubbard's U). This is related to the band narrowing caused by the antiferromagnetism. The small gap insulating ground state, obtained in the magnetic GGA calculations agrees with a semiconducting temperature dependence of the electric resistivity, observed in Ref. 4

\section{CORRELATION EFFECTS}

It is well known that for the description of the electronic and magnetic properties of the transition metal oxides one often needs to take into account strong Coulomb correlations, which can be incorporated in the calculation scheme via the $\mathrm{LDA}+\mathrm{U} / \mathrm{GGA}+\mathrm{U}$ [8] or LDA+DMFT [16] formalism.

We repeated the total energy calculation for some of the magnetic configurations within the GGA+U approx- 
TABLE II: The total energies of the different magnetic solutions, as a result of the GGA+U calculations in the PWscf code. Total energies are given per formula unit. The notations are the same as in Tab. [

\begin{tabular}{c|c|c}
\hline \hline & $\begin{array}{c}\text { Total energy } \\
(\mathrm{meV})\end{array}$ & $\begin{array}{c}\text { Band gap } \\
(\mathrm{eV})\end{array}$ \\
\hline$\uparrow m \downarrow-\uparrow m \downarrow$ & 0 & $0.26 \mathrm{eV}$ \\
$\uparrow m \uparrow-\uparrow m \uparrow$ & 18.3 & Metal \\
$\uparrow m \downarrow-\downarrow m \uparrow$ & 19.8 & $0.09 \mathrm{eV}$ \\
$\uparrow m \uparrow-\downarrow m \downarrow$ & 86.4 & Metal \\
$0 m 0-0 m 0$ & 284.6 & Metal \\
\hline \hline
\end{tabular}

imation (as mentioned above, we took the values $\mathrm{U}=3 \mathrm{eV}$ and $\mathrm{J}_{H}=0.7 \mathrm{eV}$, obtained in Ref. 3 ) and found that the account of the Coulomb correlations increases the band gap values, but does not basically change the order of the total energies for different magnetic configurations (compare Tab. I and II). This confirms that the origin of the unconventional magnetic properties is not related to the correlation effects, typical for the localized electrons, but is largely due to two factors discussed above: the formation of molecular orbitals on $\mathrm{Ru}$ trimers and charge redistribution, with the increase of d-electron number on the middle ruthenium, $\mathrm{Ru}_{m}$.

\section{SUMMARY}

Summarizing, on the basis of ab-initio and model calculations we obtained the explanation of the unusual magnetic properties of $\mathrm{Ba}_{4} \mathrm{Ru}_{3} \mathrm{O}_{10}$, which consist of the $\mathrm{Ru}$ trimers coupled via corner-shared oxygens. The ground state is found to be an antiferromagnetic insulator, with antiferromagnetic ordering both within and between $\mathrm{Ru}$ trimers. The most surprising fact - the nonmagnetic nature of the middle $\mathrm{Ru}$ ions in each trimer is explained to be a result of a combined action of the formation of molecular orbitals in the $\mathrm{Ru}$ trimers and of charge redistribution, with the increasing $d$-states occupation on the middle $\mathrm{Ru}$ which is sandwiched between two other $\mathrm{Ru}$ ions with antiparallel spins. These unusual magnetic properties of $\mathrm{Ba}_{4} \mathrm{Ru}_{3} \mathrm{O}_{10}$ are well explained by the band picture, and the electron correlations do not play significant role in the formation of this magnetic state.

\section{ACKNOWLEDGMENTS}

We thank Igor Mazin and Zlata Pchelkina for the fruitful discussions about band magnetism. This work is supported by the Russian Foundation for Basic Research via RFFI-10-02-96011 and RFFI-10-02-00140, by the Ural branch of Russian Academy of Science through the young-scientist program, the Ministry of education and science of Russia (grant 12.740.11.0026), by the German projects SFB 608, DFG GR 1484/2-1, FOR 1346, and by the European network SOPRANO.
[1] K. D. Nelson, Z. Q. Mao, Y. Maeno, and Y. Liu, Science (New York, N.Y.) 306, 1151 (2004), ISSN 1095-9203, URL http://www.ncbi.nlm.nih.gov/pubmed/15539595

[2] E. Gorelov, M. Karolak, T. O. Wehling, F. Lechermann, A. I. Lichtenstein, and E. Pavarini, Phys. Rev. Lett. 104, 225401 (2010), ISSN 0031-9007, URL http://link.aps.org/doi/10.1103/PhysRevLett.104.226401

[3] S. Lee, J.-G. Park, D. Adroja, D. Khomskii, S. Streltsov, K. A. McEwen, H. Sakai, K. Yoshimura, V. I. Anisimov, D. Mori, et al., Nature materials 5, 471 (2006), ISSN 1476-1122, URL http://www.ncbi.nlm.nih.gov/pubmed/16699512

[4] Y. Klein, G. Rousse, F. Damay, F. Porcher, G. André, and I. Terasaki, Physical Review B 84, 054439 (2011), ISSN 1098-0121, URL http://link.aps.org/doi/10.1103/PhysRevB.84.054439

[5] C. Dussarrat, F. Grasset, R. Bontchevb, and J. Darriet, Journal of Alloys and Compounds 233, 15 (1996).

[6] P. Giannozzi, S. Baroni, N. Bonini, M. Calandra, R. Car, C. Cavazzoni, D. Ceresoli, G. L. Chiarotti, M. Cococcioni, I. Dabo, et al., J. Phys.: Condens. Matter 21, 395502 (2009).

[7] J. Perdew, K. Burke, and M. Ernzerhof, Phys.
Rev. Lett. 77, 3865 (1996), ISSN 1079-7114, URL http://www.ncbi.nlm.nih.gov/pubmed/10062328,

[8] V. Anisimov, F. Aryasetiawan, and A. Lichtenstein, J. Phys.: Condens. Matter 9, 767 (1997), URL http://iopscience.iop.org/0953-8984/9/4/002

[9] O. K. Andersen and O. Jepsen, Phys. Rev. Lett. 53, 2571 (1984), URL http://link.aps.org/doi/10.1103/PhysRevLett.53.2571

[10] S. V. Streltsov, A. S. Mylnikova, A. O. Shorikov, Z. V. Pchelkina, D. I. Khomskii, and V. I. Anisimov, Phys. Rev. B 71, 245114 (2005), ISSN 1098-0121, URL http://link.aps.org/doi/10.1103/PhysRevB.71.245114

[11] R. D. Shannon, Acta Crystallographica Section A 32, 751 (1976), URL http://dx.doi.org/10.1107/S0567739476001551.

[12] I. Leonov, A. N. Yaresko, V. N. Antonov, M. A. Korotin, and V. I. Anisimov, Phys. Rev. Lett. 93, 146404 (2004), URL http://link.aps.org/doi/10.1103/PhysRevLett.93.146404

[13] E. A. Owen and E. W. Roberts, Zeitschrift fuer Kristallographie, Kristallgeometrie, Kristallphysik, Kristallchemie 96, 497 (1937).

[14] J. B. Goodenough, Magnetism and the Chemical Bond 
(Interscience-Wiley, 1963).

[15] I. I. Mazin and D. J. Singh, Physical Review B 56, $2556 \quad$ (1997), URL http://prb.aps.org/abstract/PRB/v56/i5/p2556_1
[16] V. I. Anisimov, A. I. Poteryaev, M. A. Korotin, A. O. Anokhin, and G. Kotliar, J. Phys. Cond. Matt. 9, 7359 (1997). 